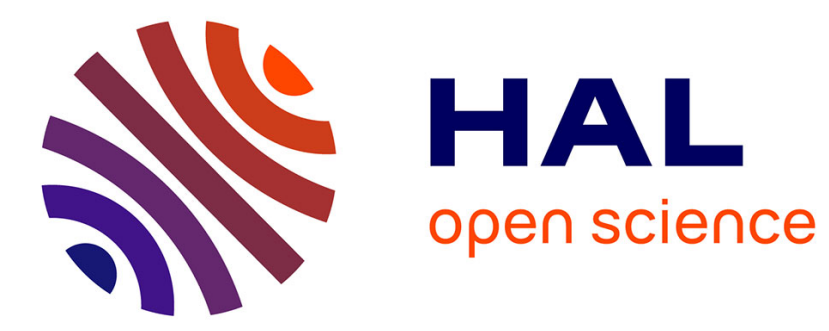

\title{
Quelques conséquences politiques de la distinction entre capitalisme et marché
}

Serge Walery

\section{To cite this version:}

Serge Walery. Quelques conséquences politiques de la distinction entre capitalisme et marché. L'Économie politique, 2008, 2008/4 (40), pp.101-112. 10.3917/leco.040.0101 . hal-01224312

\section{HAL Id: hal-01224312 \\ https://hal.science/hal-01224312}

Submitted on 4 Nov 2015

HAL is a multi-disciplinary open access archive for the deposit and dissemination of scientific research documents, whether they are published or not. The documents may come from teaching and research institutions in France or abroad, or from public or private research centers.
L'archive ouverte pluridisciplinaire HAL, est destinée au dépôt et à la diffusion de documents scientifiques de niveau recherche, publiés ou non, émanant des établissements d'enseignement et de recherche français ou étrangers, des laboratoires publics ou privés. 


\section{Quelques conséquences politiques de la distinction économique du capitalisme et du marché}

Après des décennies durant lesquelles le renouveau libéral a soigneusement entretenu la confusion du capitalisme et du marché, on a pu récemment observer un renouvellement des réflexions et contributions visant à remettre un peu de rigueur logique dans cette confusion. Le récent numéro de L'Économie Politique, ${ }^{1}$ consacré à l'économie de marché, en est une illustration.

C'est dans le cadre de ce renouvellement que, dans un précédent article de L'Économie Politique, ${ }^{2}$ on s'était attaché à montrer comment, dès ses origines, le capitalisme s'est nettement distingué du marché, que celui-ci soit appréhendé d'un point de vue purement théorique, celui de l'univers walrassien et des lois de la concurrence parfaite, ou d'un point de vue historique, c'est à dire comme l'ensemble des conditions et modalités concrètes de l'échange monétaire (entendu comme point de "jonction" de l'offre et de la demande), en un "instant" et en un "lieu" donnés. On montrait, pour conclure, que l'assimilation aujourd'hui très répandue du capitalisme et du marché, parce qu'elle nie I'histoire, ${ }^{3}$ interdit de penser une alternative au capitalisme. Comme l'a depuis souligné Marc Fleurbaey4: "l'expression "économie de marché" a remplacé le mot "capitalisme". (...) C'est regrettable, car en le remplaçant, dans le langage courant par "économie de marché", on a accrédité l'idée pas du tout innocente que toute économie où le marché et le secteur privé ont une place centrale est analogue à ce que l'on connaît actuellement.»

Après s'être attaché à rappeler et à souligner la claire distinction du capitalisme et du marché, on se propose désormais d'en développer quelques implications d'ordre méthodologique et politique. II s'agit, d'une part, de repérer les répercussions logiques et méthodologiques que cette distinction économique induit, dans la perspective de l'élaboration d'une pensée critique du capitalisme contemporain ; d'autre part, de montrer en quoi elle conduit à reconsidérer et à nuancer certains clivages, qui aujourd'hui affaiblissent la pensée et l'action critiques.

En développant brièvement la distinction précédemment établie ${ }^{5}$ entre le marché théorique (celui de l'univers walrassien), et le marché historique (l'ensemble des modalités de l'échange monétaire en un temps et en un espace économique donnés), on rappellera, dans un premier temps, que le marché théorique étant une utopie logico-déductive, son invocation ne saurait se substituer à l'analyse de la réalité concrète du marché historique.

On observera ensuite qu'en ignorant méthodologiquement cette évidence et en se focalisant sur la dénonciation du marché théorique, la plupart des pensées prétendument critiques sur le capitalisme se trompent de cible et s'invalident ainsi elles-mêmes. À l'inverse, assumer logiquement cette évidence conduit à déplacer l'objet de l'analyse, et permet d'esquisser un cadre dans lequel inscrire une pensée critique du capitalisme qui ne se trompe pas de cible.

\footnotetext{
${ }^{1}$ Qu'est-ce-que l'économie de marché ? L’Économie Politique, n`37, janvier 2008.

${ }^{2}$ Serge WALERY. Capitalisme et marché à la Renaissance. L’Économie Politique, n³0, avril 2006, pp 87-112.

3 "Assimiler capitalisme et marché procède d'une position abusivement dogmatique qui ne rend pas justice à la richesse des expériences historiques de marché. "Roger GUESNERIE. L'économie de marché. Paris, Éditions le Pommier, 2006, p 28.

${ }^{4}$ Marc FLEURBAEY. Capitalisme ou démocratie? L'alternative au $21^{\text {ème }}$ siècle. Paris, Grasset, Nouveau Collège de Philosophie, 2006, pp 12-13.

${ }^{5}$ Serge WALERY (2006). Op cit.
} 
On remarquera enfin qu'assumer méthodologiquement la claire distinction du capitalisme et du marché modifie le regard qu'on peut porter sur certains clivages politiques établis, et conduit, en particulier, à insister sur les complémentarités plutôt que sur les contradictions, entre les perspectives réformiste et alternative.

\section{Marché théorique et marché historique}

II convient, en premier lieu, de rappeler une évidence reconnue par tous, mais curieusement, sitôt oubliée dans bien des raisonnements: "il est assurément vain de chercher un marché autorégulateur qui n'existe dans aucune société. " ${ }^{6}$ Jusqu'à ce jour, dans aucune société humaine, passée ou présente, n'a existé ou existe un système de marchés un tant soit peu conforme aux lois théoriques, à l'univers walrassien. Le marché théorique n'a jamais eu de réalité historique.

On le réalise aisément concernant les sociétés non marchandes ${ }^{7}$, et toutes les sociétés au sein desquelles les activités marchandes n'ont pas atteint l'échelle suffisante (en termes d'espace, de monnaie, de quantités ou de nombre d'agents...) pour qu'émerge un système de marchés ressemblant de près ou de loin à celui que définissent les lois théoriques. Mais sans doute est-il nécessaire de rappeler que le marché théorique est tout aussi introuvable au sein des sociétés de l'ère moderne, y compris dans les périodes durant desquelles les préceptes libéraux semblent avoir été les plus influents.

Ainsi, la première moitié du 19ème siècle, ou plus précisément la période séparant la fin des systèmes corporatifs de la montée en puissance du mouvement ouvrier, est généralement considérée, par les économistes-historiens, comme celle de l'apogée de l'influence concrète des principes libéraux et en particulier de la concurrence. On note cependant que si cette influence semble avoir alors été significative en matière d'organisation du rapport salarial, ce fut déjà moins le cas en matière de concurrence entre firmes, et encore moins dans le champ des échanges internationaux 8 ; il faudra attendre la seconde moitié du 19ème siècle, pour que les principes libre-échangistes commencent lentement à être très partiellement mis en oeuvre. Surtout, même si le marché du travail issu de la dissolution des systèmes corporatifs semble avoir été, pendant quelques décennies, relativement atomisé et peu réglementé, il n'en était pas moins très éloigné de l'idéal théorique. Qui peut décemment affirmer que les prolétaires de la première moitié du 19 ème siècle ressemblaient en quoi que ce soit à des individus, libres et indépendants, disposant de dotations de survie leur permettant d'arbitrer entre l'utilité du salaire et la désutilité du temps de travail, et opérant des choix rationnels sur la base de leurs préférences individuelles ? Faut-il rappeler les difficultés et la durée de ce que les historiens ont appelé la "disciplinarisation des classes laborieuses" (y compris avec le soutien de la réglementation, comme dans le cas des "working houses" anglaises), ou la sur-mortalité considérable qui affectait alors le

\footnotetext{
${ }^{6}$ Christophe PÉBARTHE. La cité et le marché. Revue de Synthèse, $5^{\text {ème }}$ série, tome $127,2^{\text {ème }}$ semestre 2006, pp 453-458.

${ }^{7}$ Sociétés au sein desquelles il n'existe pas d'échanges marchands, c'est à dire d'échanges fondés sur un rapport quantitatif. Voir, à titre d'exemple, le partage du produit de la chasse chez les Guayaki : Pierre CLASTRES. Chronique des indiens Guayaki. Paris, Plon, Terre Humaine, 1972.

8 Sur ce thème, voir: Jean-Pierre HIRSCH. La concurrence : discours et pratiques, hier et aujourd'hui. L'Économie Politique, n³7, janvier 2008, pp 66-76. "Dès ces temps de "capitalisme pur",(...) le libéralisme ambiant n'empêche pas les opérateurs les plus efficaces de jouer de la diversité des échelles et d'une panoplie où la relation de concurrence n'est qu'une relation parmi d'autres. ”
} 
prolétariat. ${ }^{9}$ Considérer une telle configuration comme conforme à l'idéal du marché théorique serait faire insulte à son incarnation, Léon Walras ! Au cours de cette période privilégiée d'influence libérale, la matérialisation des lois théoriques du marché apparaît ainsi très partielle, aussi bien du fait que les différentes composantes de l'organisation économique sont très inégalement concernées, que parce que là où l'influence libérale se concrétise, elle ne tend à la mise en œuvre que d'une partie des lois définissant le marché théorique. On est donc bien loin d'un système complet de marchés, de l'idéal walrassien, de l'Équilibre Général.

L'irréalisme historique du marché théorique ne devrait d'ailleurs surprendre personne, dans la mesure où toute la pensée économique moderne considère, certes plus ou moins explicitement, le marché théorique comme une impossibilité. C'est, en premier lieu, le cas du "créateur" de l'Équilibre Général lui-même, dont tous les étudiants débutant leurs études d'économie apprennent, depuis des décennies, que sa démarche ne relevait pas de l'économie positive mais de l'économie normative, qu'elle ne visait pas à rendre compte de la réalité mais à caractériser un état optimal, idéal. Ces mêmes étudiants apprennent ensuite que Kenneth Arrow et Gérard Debreu ont démontré, à travers les théorèmes de l'économie du bien-être, que, dès lors qu'existent des biens collectifs, des externalités et des rendements croissants, cet idéal n'était même plus une garantie d'optimalité. Moins directement, mais beaucoup plus concrètement, la pensée contemporaine, dans son foisonnement (information et concurrence imparfaites, rigidités, interactions stratégiques, économie expérimentale, nouvelle économie institutionnelle, économie des conventions...), nous montre quotidiennement à quel point, tout effort de prise en compte, même très partielle, de la réalité, nous éloigne irrémédiablement de l'univers walrassien, du marché théorique. ${ }^{10}$

Le marché théorique, symbolisé par l'Équilibre Général walrassien, apparaît ainsi comme une utopie logico-déductive, au sens étymologique du mot utopie. II ne s'agit pas tant de taxer le marché théorique d'organisation sociale chimérique, que de constater que le marché théorique ne s'inscrit concrètement en aucun lieu, à quoi il faudrait ajouter, ni en aucune époque. Dans une perspective historique, il n'a jamais existé ; dans une perspective théorique, il ne peut pas exister.

Dès lors, si le marché théorique, cette « construction théologique, purement interne au monde des idées ${ }^{11}$, peut contribuer, par l'éclairage et le point de vue très spécifiques qu'il apporte, à mieux faire ressortir certains aspects particuliers du marché concret, la pente "naturelle" conduisant à en faire l'outil (le prisme) privilégié, voire exclusif, d'appréhension de la réalité économique contemporaine est une impasse logique.

À l'inverse de l'utopie du marché théorique, le marché concret est, lui, par définition, matériellement inscrit dans l'espace et dans le temps; il est le "lieu", ainsi que l'ensemble des conditions et modalités concrètes de l'échange monétaire, lesquelles ne cessent de se transformer, et

\footnotetext{
${ }^{9}$ Par exemple, une enquête portant sur les enfants des ouvriers des filatures de coton de la région de Mulhouse nés entre 1823 et 1834 , a montré que $27 \%$ d'entre eux ont atteint l'âge de 10 ans, $17 \%$ ont atteint 20 ans, $6 \%$ ont atteint 40 ans, et $1 \%$ a dépassé 60 ans.

Voir : Pierre JACCARD. Histoire sociale du travail de l'Antiquité à nos jours. Paris, Payot, 1960.

10 "Le marché à la Walras, celui d'une concurrence parfaite, avec un prix d'équilibre résultant d'une offre déterminée par les coûts et d'une demande déterminée par les préférences, est une fiction.» Denis CLERC. Les principes théoriques du marché. L'Économie Politique, n³7, janvier 2008, pp 737.

11 Michel AGLIETTA. Régulation et crises du capitalisme; l'expérience des États-Unis. Paris. Calmann-Levy. 1976.
} 
peuvent prendre des formes extrêmement variées. ${ }^{12}$ Le marché concret, la façon dont se rencontrent et s'articulent la production et la consommation, apparaît comme la résultante, à un instant donné, des contraintes de toutes sortes (techniques, géographiques, réglementaires, institutionnelles, conventionnelles, comportementales...) et des dynamiques sociales qui les produisent et y sont confrontées. ${ }^{13}$ "Loin d'être des faits naturels, les marchés sont le fruit d'évolutions sociales et historiques complexes (...). En ce sens, ils constituent des constructions sociales et politiques, (...) qui prennent des formes extrêmement diverses, très éloignées de la vision du marché walrassien pur. ${ }^{14}$ "

Dans toute société (espace-temps) au sein de laquelle existe un système "significatif" d'économie de marché, d'échanges marchands monétaires, le marché concret, c'est celui que définissent ce que nous avons appelé les lois historiques du marché ${ }^{15}$; le marché concret, c'est le marché historique.

\section{Le libéralisme, trompe-l'œil du capitalisme}

S'il demeure malheureusement nécessaire de rappeler de telles évidences, alors qu'en tant que telles, elles sont presque unanimement admises, c'est que, paradoxalement, leurs répercussions méthodologiques sont bien rarement prises en compte ; comme si, une fois admises, elles étaient sitôt oubliées. Le marché théorique continue ainsi de constituer l'instrument privilégié voire exclusif d'appréhension de la réalité économique auquel recourent, aussi bien ceux qui prétendent contribuer à l'analyse et à la compréhension du capitalisme contemporain, que ceux qui cherchent à en élaborer une pensée critique ou alternative. Cependant, faire du marché théorique la référence centrale ou le prisme privilégié de l'analyse de la réalité économique est source d'un biais méthodologique invalidant. Une telle démarche revient en effet à postuler, et presque toujours de façon inconsciente, que le marché théorique est ce vers quoi tend, ou s'efforce de tendre, le capitalisme. Or, comme on l'a précédemment montré, ${ }^{16}$ et comme ne cessait de le rappeler Fernand Braudel, ${ }^{17}$ ce postulat est un contre-sens historique ; le capitalisme n'a jamais concrètement promu le marché théorique dans son ensemble, bien au contraire.

12 Roger GUESNERIE (2006, Op cit, p 25) souligne ainsi «l'immense variété historique des économies de marché »

${ }^{13}$ Sur ce thème, voir : Serge WALERY. Les Hommes d'Affaires de la Renaissance et I'"esprit du capitalisme" : l'exemple de la lettre de change. Actes du Forum de la Régulation 2003, octobre 2003.

${ }^{14}$ Benjamin CORIAT, Olivier WEINSTEIN. La construction sociale des marchés. La Lettre de la Régulation, $n^{\circ} 53$, septembre 2005.

Sur ce thème, voir également: Laure BAZZOLI, Véronique DUTRAIVE. La conception institutionnaliste du marché comme construction sociale : une économie politique des institutions. In : Guy BENSIMON (coord). Histoire des représentations du marché. Paris, Michel Houdiard Éditeur, 2005, pp 670-691.

${ }^{15}$ Serge WALERY (2006). Op cit.

${ }^{16}$ Serge WALERY (2006). Op cit.

${ }^{17}$ Voir, par exemple :

- Fernand BRAUDEL. Civilisation matérielle, économie et capitalisme. Paris, Armand Colin, 1979, Tome 3, p 537-539.

- Fernand BRAUDEL. La dynamique du capitalisme. Paris, Arthaud, 1985, pp 118-119. 
La plus grande part des discours sur le capitalisme contemporain repose pourtant, le plus souvent implicitement, sur ce postulat. En faisant consciemment ou inconsciemment du marché théorique le prisme privilégié voire exclusif de leur observation du capitalisme, alors qu'il s'agit d'un prisme particulièrement déformant, ces discours se condamnent à de graves illusions d'optique.

C'est particulièrement le cas des pensées et discours à visée critique, pour lesquels ce biais méthodologique affecte gravement la cohérence logique des raisonnements développés, et génère des résultats contre-productifs. Dans une telle perspective, la confusion du capitalisme et du marché théorique revient en effet à critiquer une utopie logico-déductive, en croyant élaborer une pensée critique de la réalité historique et sociale. Certes, critiquer une utopie logico-déductive en tant que telle n'est pas dénué d'intérêt. Mais le faire en s'imaginant développer une analyse critique de la réalité historique conduit, non seulement à se tromper de cible, mais aussi, et de façon totalement contreproductive, à démontrer l'extraordinaire efficacité idéologique de ladite utopie. ${ }^{18}$ C'est ainsi que la plupart de ceux qui prétendent s'attacher à critiquer le capitalisme contemporain, voire à imaginer une possible alternative, consacrent de fait l'essentiel de leur énergie, à s'attaquer à l'utopie du marché théorique que les thuriféraires du capitalisme agitent, tel un chiffon, pour capter leur attention.

C'est pourquoi, tenter d'appréhender le capitalisme contemporain suppose, en premier lieu, de rompre avec son assimilation, consciente ou non, au marché théorique. Comme on l'a vu, ${ }^{19}$ si le développement capitaliste pèse très lourdement sur l'organisation concrète du marché, ce n'est aucunement en s'efforçant d'inscrire dans la réalité l'ensemble des lois théoriques du marché, mais en promouvant, en fonction de la réalité historique du moment, les lois historiques les plus propices à l'accumulation, quand bien même celles-ci entrent en contradiction directe avec celles-là. Et si la logique générale des lois historiques du marché promues par le développement capitaliste demeure invariablement l'accumulation, les principes et les modalités concrètes de sa mise en œuvre s'adaptent sans cesse à l'évolution des contraintes (techniques, géographiques, réglementaires, institutionnelles, conventionnelles, comportementales...) et des dynamiques sociales. Dès lors, appréhender le capitalisme contemporain et ses relations avec le marché passe nécessairement par le repérage et l'analyse des lois historiques du marché que le développement actuel du capitalisme tend à concrétiser. ${ }^{20}$

Sans doute de longs développements sont-ils superflus, pour souligner à quel point le marché historique est, aujourd'hui, déterminé/conditionné par les impératifs de l'accumulation. II existe même un accord général pour constater que, depuis l'épuisement du mode de développement fordiste, l'évolution des relations et des rôles respectifs de l'actionnaire, du manager et du salarié, les mutations des firmes, la globalisation et le poids croissant des marchés financiers, sont autant de manifestations concrètes du renforcement de l'influence des impératifs de l'accumulation dans l'évolution des lois historiques du marché, aux dépens d'autres dynamiques sociales (celle des États-nations ou celle du salariat, par exemple). Et au delà des discours incantatoires sur l'idéal du marché théorique, qui peut croire un seul instant que la dynamique actuelle du capitalisme nous rapproche de cet idéal, alors que les secteurs les

\footnotetext{
${ }^{18}$ Sur la dimension idéologique de l'assimilation du capitalisme et du marché théorique, voir : Serge WALERY (2006). Op cit.

${ }^{19}$ Serge WALERY (2006). Op cit.

${ }^{20}$ On peut souligner à cet égard que les historiens semblent beaucoup plus convaincus que les économistes de la nécessité "d'abandonner le marché walrassien et de réfléchir à la construction sociale et politique des marchés, autrement dit au marché institué » [Christophe PÉBARTHE (2006). Op cit], et "d'historiciser la catégorie de marché, à rebours de toute abstraction intemporelle, de tout schéma universel préconstruit ». Dominique MARGAIRAZ, Philippe MINARD. Le marché dans son histoire. Revue de Synthèse, $5^{\text {ème }}$ série, tome $127,2^{\text {ème }}$ semestre 2006, pp 241-252.
} 
plus prometteurs sont presque tous à rendements croissants, que les pratiques anticoncurrentielles des firmes, des intermédiaires financiers et des États sont la règle, ou que le pouvoir de marché des fonds d'investissement et des grandes firmes ne cesse de se renforcer?

Dès lors qu'on renonce à appréhender le capitalisme à travers l'utopie du marché théorique et qu'on s'efforce de l'envisager comme une dynamique sociale visant à soumettre et adapter les lois historiques du marché aux impératifs sans cesse renouvelés de l'accumulation du capital, l'objet de l'analyse se "déplace". Pour qui prétend exercer un regard critique sur le capitalisme contemporain, il ne s'agit plus de focaliser sa réflexion et son énergie sur ce trompe-l'œil que constitue le discours libéral, mais sur ce que la dynamique du capitalisme met concrètement en œuvre pour adapter le marché historique aux impératifs de l'accumulation.

\section{Réforme vs alternative : contiguïté théorique et continuum temporel}

Remettre ainsi "à l'endroit" l'analyse économique du capitalisme contemporain n'est évidemment pas sans conséquences d'ordre politique. Cela conduit inévitablement à modifier le regard qu'on peut porter sur les débats et divergences qui structurent le champ politique actuel, et en particulier sur le clivage entre réformistes et alternatifs. C'est, en effet, dans l'assimilation du capitalisme et du marché théorique que ce clivage trouve sa source.

Dès lors que le capitalisme est assimilé au marché théorique, les débats se structurent naturellement autour de la question binaire de l'acceptation dudit marché, couramment désigné dans ce cadre par le terme d'économie de marché. C'est ainsi que naît le clivage entre réformistes, acceptant l'économie de marché, et alternatifs, la refusant plus ou moins explicitement. Et s'il existe, bien sûr, quantités de degrés possibles dans la réponse à une telle question, le clivage n'en demeure pas moins irréductible.

À l'inverse, si on s'affranchit de l'utopie du marché théorique, et que l'analyse et la critique se portent sur les lois historiques du marché que promeut concrètement la dynamique contemporaine du capitalisme dans sa course sans fin à l'accumulation, les perspectives réformiste et alternative apparaissent plus contiguës, voire complémentaires, que contradictoires.

La dynamique du capitalisme tendant à soumettre les lois historiques du marché aux seuls besoins et impératifs (en permanente redéfinition) de l'accumulation du capital, opter pour une perspective réformiste consiste à considérer que les effets pervers de cette dynamique sont trop importants pour qu'on lui laisse le champ libre. II convient alors d'œuvrer à la prise en compte d'autres impératifs dans la définition des lois historiques du marché ; les uns insisteront sur la prise en compte d'impératifs sociaux, ${ }^{21}$ d'autres sur les impératifs écologiques, d'autres encore sur les impératifs démocratiques. ${ }^{22}$ Par ailleurs, il existe des nuances importantes depuis ceux qui, globalement, apprécient positivement la dynamique du capitalisme mais considèrent indispensable de lutter contre ses inévitables effets pervers, jusqu'à ceux qui, fondamentalement critiques à l'égard du capitalisme, n'en acceptent pas moins sa prépondérance historique. Cependant, au delà de cette variété, la perspective réformiste consiste à promouvoir d'autres impératifs que ceux de l'accumulation dans la définition des lois historiques du marché, c'est à dire à encadrer, ou plus précisément, à canaliser la dynamique du capitalisme.

Dans une perspective alternative, les effets pervers de la dynamique du capitalisme apparaissent tels, qu'il est illusoire d'essayer de la canaliser, et qu'elle doit être remise en cause en tant

\footnotetext{
${ }^{21}$ Pauvreté, exclusion, inégalités de développement...

${ }^{22}$ On pense ici à des démarches telles que celle d'Amartya Sen.
} 
que telle. II convient alors de concevoir une logique générale susceptible de se substituer à terme à celle de l'accumulation, et d'œuvrer pour que ses impératifs s'imposent dans la définition et l'évolution des lois historiques du marché. Plus globalement, il s'agit donc de promouvoir une dynamique sociale susceptible de se substituer à terme à celle du capitalisme, en tant que dynamique dominante ou prépondérante. Le simple énoncé de cette perspective en montre l'extrême ambition. C'est pourquoi, au delà même du contresens logique et historique que constitue l'assimilation du capitalisme et du marché, on ne peut être que dubitatif face à ceux qui jugent utile d'en rajouter, en prétendant non seulement rompre avec la dynamique du capitalisme, mais en outre en finir avec le marché. ${ }^{23}$

Ainsi, même en distinguant clairement le capitalisme du marché, les perspectives réformiste et alternative n'en demeurent pas moins profondément différentes, et à long terme, divergentes. Dans le premier cas, il s'agit de canaliser la dynamique du capitalisme et donc, au moins implicitement, d'en accepter l'existence, voire la prépondérance ; dans le second, l'objectif de long terme est bien de substituer une autre dynamique sociale à celle du capitalisme, et donc d'en finir avec celle-ci. Pour autant, ces deux perspectives se trouvent confrontées à un même problème de moyens, et sur le plan théorique comme sur le plan temporel, la frontière qui les sépare se révèle progressive, graduelle.

À court et moyen terme, dans une perspective réformiste comme dans une perspective alternative, l'objectif est de faire prévaloir d'autres impératifs que ceux de l'accumulation dans la définition des lois historiques du marché. Certes, et contrairement aux réformistes, les alternatifs le font au nom d'une logique générale censée se substituer à terme à celle de l'accumulation. Cependant, dans un cas comme dans l'autre, à court et moyen terme, la question des moyens se pose de la même façon : face à la puissance actuelle de la dynamique de l'accumulation et à la prépondérance de ses impératifs, quelles sont les forces sociales susceptibles de porter la prise en compte d'autres impératifs ? Le moins que l'on puisse dire est que, dans l'état actuel des forces sociales susceptibles de soutenir l'une ou l'autre perspective, on a peine à imaginer que réformistes et alternatifs puissent avoir la moindre chance de succès en se livrant à une concurrence acharnée en la matière.

Et ce, d'autant plus que, dès lors qu'on cesse d'assimiler capitalisme et marché, la frontière entre perspective réformiste et perspective alternative cesse d'être définie par la réponse à la question binaire de l'acceptation de l'économie de marché, et devient ainsi beaucoup moins nette qu'il y paraît. Entre définir et promouvoir la prise en compte d'autres impératifs que ceux de l'accumulation, et définir une logique générale et en promouvoir les impératifs, il y a certes divergence de perspectives, mais pas de contradiction logique ni d'incompatibilité concrète. ${ }^{24}$

Dans la mesure où une logique est le fondement d'un ensemble d'impératifs en permanente adaptation aux évolutions de la réalité sociale, la réflexion sur la logique et celle sur les impératifs ne sauraient être disjointes. Que les efforts de conception et d'imagination des réformistes soient focalisés sur les impératifs, et que ceux des alternatifs le soient sur la logique n'empêche donc pas une certaine complémentarité. Ainsi, même si les réformistes ne se fixent pas explicitement comme objectif de substituer une autre logique à celle de l'accumulation, les impératifs qu'ils s'efforcent de promouvoir ne peuvent être pensés ex nihilo. Ils relèvent, par nature, d'une autre "source d'inspiration", c'est-à-dire d'une autre logique que celle de l'accumulation, fut-elle implicite, indéterminée voire inconsciente. En outre, même si les impératifs (précédemment évoqués) que promeuvent les réformistes ne le sont pas au nom d'un combat frontal contre le capitalisme, on peut imaginer que certains d'entre eux soient compatibles, voire convergents avec une logique alternative; auquel cas, à moins de privilégier la

\footnotetext{
${ }^{23}$ Voir, par exemple: Alain CAILLÉ. Dé-penser l'économique; contre le fatalisme. Paris, La Découverte/MAUSS, 2005.
}

${ }^{24}$ Sous réserve de ne plus croire au "grand soir". 
politique du pire, réformistes et alternatifs devraient, au moins temporairement et dans des domaines d'action précis, pouvoir faire converger leurs forces.

Surtout, si perspective réformiste et perspective alternative sont clairement divergentes dans une perspective de long terme, leur mise en œuvre à court et moyen terme passe, dans les deux cas, par la mobilisation de forces sociales promouvant la prise en compte d'impératifs qui ne sont pas ceux de l'accumulation. Certes, pour les alternatifs, la logique préexiste, et génère les impératifs ; ils savent cependant que sa mise en œuvre et plus encore sa prépondérance relèvent d'une perspective de long terme, et qu'il s'agit à court terme de faire prévaloir des impératifs "issus" de la logique. Les réformistes, eux, se focalisent clairement sur les impératifs susceptibles, à court et moyen terme, de contrebalancer ceux de l'accumulation; pour autant, rien ne permet d'exclure, qu'à long terme, ces impératifs, développés, réadaptés et renouvelés en fonction des évolutions concrètes, ne finissent par dessiner une logique générale susceptible de concurrencer, voire de supplanter, celle de l'accumulation. II existe ainsi, entre ces deux perspectives, un continuum temporel : la perspective réformiste apparaît plutôt comme une perspective de court terme, la perspective alternative clairement comme une perspective de long terme. De façon très simplificatrice, on pourrait dire que l'alternative, c'est la réforme dans une perspective de longue durée.

Bien entendu, pour tout individu critique à l'égard du développement capitaliste contemporain, la perspective alternative apparaît infiniment plus enthousiasmante que la perspective réformiste ; la tentation est donc grande d'ignorer le court terme pour se projeter directement dans le long terme. C'est là une configuration bien familière, pour les économistes. Comme le souligne Hervé Hamon 25 : "L'idéologie de l'économie induit une perception du temps qui privilégie la longue durée. A rebours de ce qu'affirmait Nietzsche, le court terme y apparaît comme le temps des rigidités et de l'impuissance qui ne laisse que des "marges" de manœuvre ; c'est le temps triste de l'"éternel retour du même", rythmé par les chroniques de conjoncture. Le long terme, au contraire, autorise la flexibilité, la mobilité, le changement des structures et des comportements sans lequel rien d'heureux ne saurait arriver ; c'est le temps d'exercice de la liberté et de la vie, le temps de la vérité. "

Pour autant, les économistes sont bien conscients, au moins depuis Keynes, qu'aussi attrayant le long terme soit-il, cela ne les autorise pas à s'affranchir du court terme. Et tout un chacun sait bien que, poussée à l'extrême, la tendance à s'affranchir du court terme conduit au "grand soir", autre utopie néfaste.

\section{Pour conclure}

Au terme de ces deux articles qui nous ont si rapidement menés des comportements des hommes d'affaires de la Renaissance à certains enjeux politiques contemporains, sans doute convient-il de rappeler, une dernière fois, que sans distinction claire du capitalisme et du marché, et en particulier du marché théorique, toute pensée critique du capitalisme se fourvoie, se trompe de cible.

Outre le gaspillage d'énergie et d'intelligence critiques qu'il constitue, un tel fourvoiement conduit surtout à opposer artificiellement réformistes et alternatifs, interdisant ainsi toute convergence entre ces deux forces, qui se distinguent certes par leur degré et leur perspective temporelle, mais n'en sont pas pour autant incompatibles.

Au moment où l'influence idéologique du renouveau libéral semble nettement s'essouffler, ${ }^{26}$ et alors que les difficultés et les incertitudes nous rappellent quotidiennement que le devenir du capitalisme

\footnotetext{
${ }^{25}$ Hervé HAMON. L'économie des économistes et celle de l'air du temps. L'état de la France 19992000. Paris, La Découverte, 1999, pp 59-62.

${ }^{26}$ Voir : Christian CHAVAGNEUX. Les dernières heures du libéralisme; mort d'une idéologie. Paris, Perrin, 2007.
} 
contemporain n'est aucunement tracé d'avance, on ne peut que déplorer l'attitude autodestructrice de ces réformistes et alternatifs qui entretiennent la confusion et les divergences. Puissent-ils, face à l'incertitude et aux enjeux actuels, consacrer plutôt leur énergie à dissiper la confusion et à comprendre, qu'au moins à court terme et sur certains impératifs, réformistes et alternatifs peuvent converger, et que cultiver ces convergences est une condition essentielle de leur efficacité.

Serge Walery

P.R.E.S. Aix-Marseille Université

Mars 2008 


\section{BIBLIOGRAPHIE}

Aglietta M., 1976, Régulation et crises du capitalisme ; l'expérience des Etats-Unis. Paris, Calmann-Levy, 1976.

Bazzoli L., Dutraive V., 2005, La conception institutionnaliste du marché comme construction sociale: une économie politique des institutions ». In : Guy BENSIMON (coord). Histoire des représentations du marché. Paris, Michel Houdiard Éditeur, 2005, pp 670-691.

Braudel F., 1979, Civilisation matérielle, économie et capitalisme. 3 volumes, Paris, Armand Colin, 1979.

Braudel F., 1985, La dynamique du capitalisme. Paris, Arthaud, 1985.

Caillé A., 2005, Dé-penser l'économique ; contre le fatalisme. Paris, La Découverte/MAUSS, 2005.

Chavagneux C., 2007, Les dernières heures du libéralisme ; mort d'une idéologie. Paris, Perrin, 2007.

Clastres P., 1972, Chronique des Indiens Guayaki. Paris, Plon, Terre Humaine, 1972.

Clerc D., 2008, « Les principes théoriques du marché ». L’Économie Politique, n³7, janvier 2008, pp 7-37.

Coriat B., Weinstein O., 2005, "La construction sociale des marchés». La Lettre de la Régulation, n53, septembre 2005.

Fleurbaey M., 2006, Capitalisme ou démocratie ? L'alternative au 21ème siècle. Paris, Grasset, Nouveau Collège de Philosophie.

Guesnerie R., 2006, L'économie de marché. Paris, Éditions le Pommier.

Hamon H., 1999, « L'économie des économistes et celle de l'air du temps ». In : L'état de la France 1999-2000. Paris, La Découverte, 1999, pp 59-62.

Hirsch J-P., 2008, « La concurrence: discours et pratiques, hier et aujourd'hui ». L'Économie Politique, $n^{\circ} 37$, janvier 2008, pp 66-76.

Jaccard P., 1960, Histoire sociale du travail de l'Antiquité à nos jours. Paris, Payot, 1960.

Margairaz D., Minard P., 2006, « Le marché dans son histoire ». Revue de Synthèse, 5ème série, tome 127, 2ème semestre 2006, pp 241-252.

Pébarthe C., 2006, « La cité et le marché ». Revue de Synthèse, 5ème série, tome 127, 2ème semestre 2006, pp $453-458$.

Walery S., 2003, «Les hommes d'affaires de la Renaissance et I'"esprit" du capitalisme ; l'exemple de la lettre de change ». Actes du Forum de la Régulation 2003, octobre 2003.

Walery S., 2006, « Capitalisme et marché à la Renaissance ». L’Économie Politique, n³0, avril 2006, pp 87-112. 